\title{
Low-Complexity Expectation Propagation Detection for Uplink MIMO-SCMA Systems
}

\author{
Zeina Mheich \\ 5 GIC \\ University of Surrey \\ Guildford, U.K. \\ z.mheich@surrey.ac.uk
}

\author{
Ibrahim A. Hemadeh \\ 5 GIC \\ University of Surrey \\ Guildford, U.K. \\ i.hemadeh@surrey.ac.uk
}

\author{
Zilong Liu \\ CSEE \\ University of Essex \\ Essex, U.K. \\ zilong.liu@essex.ac.uk
}

\author{
Pei Xiao \\ $5 G I C$ \\ University of Surrey \\ Guildford, U.K. \\ p.xiao@surrey.ac.uk
}

\begin{abstract}
We consider uplink sparse code multiple access (SCMA) systems associated with multiple input multiple output (MIMO), where the transmitters and the receiver are equipped with multiple antennas, for enhanced reliability (diversity gain) or improved data rate (multiplexing gain). For each diversity or multiplexing based MIMO scheme combined with SCMA, we develop low-complexity iterative detection algorithms based on the message passing algorithm (MPA) and the expectation propagation algorithm (EPA). We show that the MIMO-SCMA under EPA enjoys the salient advantage of linear complexity (in comparison to the MPA counterpart with exponential complexity) as well as enhanced error rate performances due to the MIMO transmission. We also show that the performance of EPA depends on the codebook size and the number of antennas.

Index Terms-SCMA, diversity, multiplexing, message passing algorithm (MPA), expectation propagation algorithm (EPA).
\end{abstract}

\section{INTRODUCTION}

SCMA is an emerging code domain non-orthogonal multiple access (NOMA) paradigm [1] which has attracted increasing research attention over the past years. In principle, coded bits in SCMA are mapped to multi-dimensional sparse codewords which naturally combines the bit-to-constellation modulation with the multi-user spreading. For enhanced diversity gain and/or multiplexing gain, a recent trend is to integrate SCMA with MIMO technology where multiple transmit and receive antennas are deployed. For multiple access channels (MAC), the receive antennas installed at the base-station give rise to larger diversity order and hence may be used for supporting more users with multiple access gain. The diversity-multiplexing tradeoff for MAC was studied in [2]. [3] studied a downlink MIMO-SCMA scheme, where the multiple antennas employed at the transmitter are used for user multiplexing. A diversity scheme based on Alamouti encoding [4] was proposed in [5] for downlink MIMOSCMA associated with two transmit antennas and two receive antennas. In their scheme, the combiner and the multi-user detector are performed separately at the receiver. Moreover, a multiplexing scheme was proposed for uplink MIMO-SCMA systems by relying on two transmit antennas and four receive antennas, which resulted in better user connectivity than both orthogonal frequency-division multiplexing (OFDM) and single-input single-output (SISO) SCMA. In this work, we consider diversity and multiplexing based MIMO schemes for uplink MIMO-SCMA systems. More specifically, we investigate MIMO-SCMA associated with space diversity, Alamouti encoding and multiplexing techniques. For each scheme, the equivalent factor graph is constructed and the complexity of MPA over the equivalent factor graph is analyzed. Unlike the Alamouti-based SCMA scheme proposed in [5] for downlink, the combiner and the multi-user detector may not be separated in uplink, thus a joint detector using message passing on the equivalent factor graph is performed. Furthermore, we propose an expectation propagation algorithm (EPA) based detector with linear complexity. To the best of our knowledge, our work is the first which proposes a low-complexity joint detector, based on EPA, for uplink Alamouti-based SCMA scheme. The performance of our EPA detector is analyzed for each MIMO scheme mentioned in the above with different number of receive antennas and the codebook size. Finally, we present the uncoded BER performance for all schemes, under the constraint of a fixed data rate.

Notations: We use $x, \mathbf{x}$ and $\mathbf{X}$ to represent a scalar, a vector and a matrix, respectively. The $i^{\text {th }}$ element of $\mathbf{x}$ is denoted by $x_{i}$ and $X_{i j}$ is the element in the $i^{\text {th }}$ row and $j^{\text {th }}$ column of X. $\operatorname{diag}(\mathbf{x})$ returns a diagonal matrix, where its $i^{\text {th }}$ diagonal element is $x_{i}$. The modulo operator $\bmod (a, m)$ returns the remainder after division of $a$ by $m .|x|$ returns the absolute value of $x$ if $x$ is a real number and the modulus if $x$ is complex. $\mathbf{x}^{T}$ denotes the transpose of $\mathbf{x}$ and $\mathbf{x}^{*}$ returns the complex conjugate of the elements of $\mathbf{x}$.

\section{UPLINK SISO SPARSE CODE MULTIPLE ACCESS}

\section{A. System model}

We consider the uplink SCMA system, where $J$ singleantenna users $u_{j \in \mathcal{J}}$ share $K$ resources $r_{k \in \mathcal{K}}$ (OFDM subcarriers) to communicate to a single-antenna receiver, where $\mathcal{J}$ and $\mathcal{K}$ is the sets of user and resource indices respectively. The overloading factor is defined as $\lambda=J / K$.

The SCMA encoder for user $u_{j \in \mathcal{J}}$ maps every $\log _{2} M$ coded bits to a length- $K$ vector of complex symbols $\mathbf{x}_{j}=$ $\left[x_{1 j}, x_{2 j}, \cdots, x_{K j}\right]^{T}$. The vector $\mathbf{x}_{j}$ is called SCMA codeword which belongs to a finite set of $M$ codewords $\mathcal{C}_{j}$ known as SCMA codebook. The SCMA codewords are sparse, with $N<K$ non-zero values. The SCMA structure can be represented by a factor graph $\mathcal{G}$, as depicted in Figure 1. 
The circles denote variable nodes, while the squares represent resource nodes. Each variable node represents a codeword sent by a user. A variable node corresponding to a codeword $\mathbf{x}_{j}$ is connected to a resource node $r_{k}$ by an edge iif $x_{k j} \neq 0$.

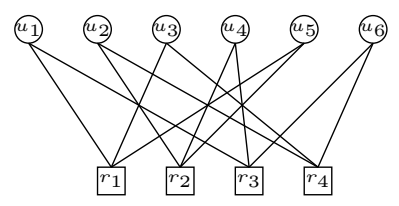

Figure 1. Factor graph representation of a regular SCMA system with 6 users and 4 resources. The overloading factor is $\lambda=150 \%$.

The received signal in the uplink can be expressed as $\mathbf{y}=\sum_{j=1}^{J} \operatorname{diag}\left(\mathbf{h}_{j}\right) \mathbf{x}_{j}+\mathbf{n}$, where $\mathbf{h}_{j}=\left(h_{1}^{j}, \cdots, h_{K}^{j}\right)^{T}$ is the channel vector between the $j$ th user and the receiver, $h_{k}^{j} \sim \mathcal{C N}(0,1)$ and $\mathbf{n}=\left(n_{1}, \cdots, n_{K}\right)^{T}$ is the complex additive white Gaussian noise $(\mathrm{AWGN}), n_{k} \sim \mathcal{C N}\left(0, N_{0}\right)$.

\section{B. Multi-user detection at the receiver}

Message passing: The MPA iteratively computes an approximation of the MAP value for each variable node in order to retrieve the estimate $\hat{\mathbf{X}}$. Let $m_{v_{j} \rightarrow r_{k}}^{(t)}(\mathbf{x})$ (resp. $m_{r_{k} \rightarrow v_{j}}^{(t)}(\mathbf{x})$ ) denotes the message associated with the codeword $\mathbf{x}$, which is transmitted by (to) the variable node $v_{j}$ to (by) the resource node $r_{k}$ at the $t$ th iteration of the MPA [1]:

$$
\begin{aligned}
m_{r_{k} \rightarrow v_{j}}^{(t)}(\mathbf{x})=\sum_{\substack{\mathbf{x}_{\mathbf{j}}=\mathbf{x} \\
i \in \mathcal{I}_{r}(k) \backslash\{j\} \\
\mathbf{x}_{i} \in \mathcal{C}_{i}}} \frac{1}{\pi N_{0}} e^{-\frac{\mid y_{k}-\sum_{\left.i \in \mathcal{I}_{r}(k) h_{k}^{i} x_{k i}\right|^{2}}}{N_{0}}} & \\
& \prod_{i \in \mathcal{I}_{r}(k) \backslash\{j\}} m_{v_{i} \rightarrow r_{k}}^{(t-1)}\left(\mathbf{x}_{i}\right),
\end{aligned}
$$

and

$$
m_{v_{j} \rightarrow r_{k}}^{(t)}(\mathbf{x})=\alpha_{j} \cdot \prod_{\ell \in \mathcal{I}_{v}(j) \backslash\{k\}} m_{r_{\ell} \rightarrow v_{j}}^{(t)}(\mathbf{x}),
$$

where $\alpha_{j}$ is a normalization factor, $\mathcal{I}_{r}(k)$ and $\mathcal{I}_{v}(j)$ are the set of variable node indices connected to resource node $r_{k}$ and the set of resource node indices connected to variable node $v_{j}$, respectively. The complexity of MP detector is $\mathcal{O}\left(K M^{d_{r}}\right)$.

Expectation propagation: In [6], an EPA was proposed for uplink SCMA systems, where only the means and variances of the messages are tracked during iterative detection on the factor graph. Furthermore, the EPA has a low detection complexity $\mathcal{O}\left(K M d_{r}\right)$, which scales linearly with the values of $M$ and $d_{r}$. The steps performed by the EPA at iteration $t$ are as follows.

1) For each variable node $v_{j \in \mathcal{J}}$, the posterior belief approximated is computed for all $\mathbf{x}_{j} \in \mathcal{C}_{j}$ as follows:

$$
q^{(t)}\left(\mathbf{x}_{j} \mid \mathbf{y}\right)=\alpha_{j} \cdot p^{(0)}\left(\mathbf{x}_{j}\right) \cdot \prod_{k \in \mathcal{I}_{v}(j)} m_{r_{k} \rightarrow v_{j}}^{(t-1)}\left(x_{k j}\right)
$$

where $\alpha_{j}$ is a normalization factor, $p^{(0)}\left(\mathbf{x}_{j}\right)$ represents the a-priori probability of codeword $\mathbf{x}_{j}$.

2) Compute the posterior mean $\mu_{k j}^{(t)}$ and variance $\zeta_{k j}^{(t)}$ for each variable node $v_{j \in \mathcal{J}}$ and resource node $r_{k \in \mathcal{I}_{v}(j)}$ as follows: $\mu_{k j}^{(t)}=\sum_{\mathbf{x}_{j} \in \mathcal{C}_{j}} q^{(t)}\left(\mathbf{x}_{j} \mid \mathbf{y}\right) \cdot x_{k j}$ and $\zeta_{k j}^{(t)}=$ $\sum_{\mathbf{x}_{j} \in \mathcal{C}_{j}} q^{(t)}\left(\mathbf{x}_{j} \mid \mathbf{y}\right) \cdot\left|x_{k j}-\mu_{k j}^{(t)}\right|^{2}$.
3) Calculate the means $\mu_{v_{j} \rightarrow r_{k}}^{(t)}$ and the variances $\zeta_{v_{j} \rightarrow r_{k}}^{(t)}$ of the messages $m_{v_{j} \rightarrow r_{k}}^{(t)} \sim \mathcal{C N}\left(\mu_{v_{j} \rightarrow r_{k}}^{(t)}, \zeta_{v_{j} \rightarrow r_{k}}^{(t)}\right)$ :

$$
\zeta_{v_{j} \rightarrow r_{k}}^{(t)}=\left(\frac{1}{\zeta_{k j}^{(t)}}-\frac{1}{\zeta_{r_{k} \rightarrow v_{j}}^{(t-1)}}\right)^{-1}, \mu_{v_{j} \rightarrow r_{k}}^{(t)}=\zeta_{v_{j} \rightarrow r_{k}}^{(t)}\left(\frac{\mu_{k j}^{(t)}}{\zeta_{k j}^{(t)}}-\frac{\mu_{r_{k} \rightarrow v_{j}}^{(t-1)}}{\zeta_{r_{k} \rightarrow v_{j}}^{(t-1)}}\right) .
$$

4) Determine the means $\mu_{r_{k} \rightarrow v_{j}}^{(t)}$ and the variances $\zeta_{r_{k} \rightarrow v_{j}}^{(t)}$ of the messages $m_{r_{k} \rightarrow v_{j}}^{(t)} \sim \mathcal{C N}\left(\mu_{r_{k} \rightarrow v_{j}}^{(t)}, \zeta_{r_{k} \rightarrow v_{j}}^{(t)}\right)$ :

$$
\begin{aligned}
& \mu_{r_{k} \rightarrow v_{j}}^{(t)}=\frac{1}{h_{k}^{j}}\left(y_{k}-\sum_{\substack{i \in \mathcal{I}_{r}(k) \\
i \neq j}} h_{k}^{i} \mu_{v_{i} \rightarrow r_{k}}^{(t)}\right), \\
& \zeta_{r_{k} \rightarrow v_{j}}^{(t)}=\frac{1}{\left|h_{k}^{j}\right|^{2}}\left(N_{0}+\sum_{\substack{i \in \mathcal{I}_{r}(k) \\
i \neq j}}\left|h_{k}^{i}\right|^{2} \zeta_{v_{i} \rightarrow r_{k}}^{(t)}\right) .
\end{aligned}
$$

The posterior LLRs are computed using the posterior beliefs approximated in (3). The EPA is initialized with $\mu_{r_{k} \rightarrow v_{j}}^{(t)}=0$ and $\zeta_{r_{k} \rightarrow v_{j}}^{(t)}=\mathrm{INF}$, where INF is a large positive constant.

\section{MIMO-SCMA: MULTIPLEXING VERSUS DIVERSITY}

Consider a MIMO-SCMA system, with $N_{t}=2$ transmit antennas and $N_{r}$ receive antennas. All users are multiplexed over blocks of OFDM subcarriers in the uplink. We study the following schemes combined with SCMA in uplink: space diversity (SD) [7], orthogonal space-time block code (OSTBC) [4] and multiplexing (MUX) [7].

Space diversity: In the context of SD-SCMA, the codeword transmitted by each user is repeated over the $N_{t}$ transmit antennas. The signal received by the $n_{r}$ th antenna is $\mathbf{y}^{n_{r}}=$ $\sum_{n_{t}=1}^{N_{t}} \sum_{j=1}^{J} \operatorname{diag}\left(\mathbf{h}_{j}^{n_{t}, n_{r}}\right) \cdot \mathbf{x}_{j}+\mathbf{n}^{n_{r}}$, where $n_{t} \in\left\{1, \cdots, N_{t}\right\}$ and $n_{r} \in\left\{1, \cdots, N_{r}\right\}$ are the transmit and receive antenna indices respectively. $\mathbf{h}_{j}^{n_{t}, n_{r}}=\left(h_{1}^{n_{t}, n_{r}, j}, \cdots, h_{K}^{n_{t}, n_{r}, j}\right)^{T}$ is the channel vector between the $n_{t}$ th transmit antenna of user $j$ and the $n_{r}$ th receive antenna, given that $h_{k}^{n_{t}, n_{r}, j} \sim \mathcal{C N}(0,1)$, while $\mathbf{n}^{n_{r}}=\left(n_{1}^{n_{r}}, \cdots, n_{K}^{n_{r}}\right)^{T}$ is the complex AWGN at the $n$th receive antenna, $n_{k}^{n_{r}} \sim \mathcal{C N}\left(0, N_{0}\right)$.

OSTBC: In the OSTBC scheme, two data symbols $s_{1}$ and $s_{2}$ are transmitted by two transmit antennas $\left(N_{t}=2\right)$ over two channel use periods $(T=2)$. It can be characterized by its encoding matrix of size $N_{t} \times T$ :

$$
\mathbf{S}=\left[\begin{array}{cc}
-s_{2}^{*} & s_{1} \\
s_{1}^{*} & s_{2}
\end{array}\right]
$$

OSTBC-SCMA is equivalent to replacing $s_{1}$ and $s_{2}$ in (6) by SCMA codewords for each user. The received signal by the $n_{r}$ th antenna at the time slot $t \in\left\{t_{0}, t_{0}+1\right\}$, is given by:

$$
\mathbf{y}^{n_{r}}(t)=\sum_{n_{t}=1}^{N_{t}} \sum_{j=1}^{J} \operatorname{diag}\left(\mathbf{h}_{j}^{n_{t}, n_{r}}\right) \cdot \mathbf{x}_{j}\left(n_{t}, t\right)+\mathbf{n}^{n_{r}},
$$

where $\mathbf{x}_{j}\left(n_{t}, t\right)$ is the codeword transmitted by user $u_{j}$ from the $n_{t}$ th transmit antenna at time $t$. The following holds:

$$
\mathbf{x}_{j}\left(n_{t}, t_{0}+1\right)=\left\{\begin{array}{r}
-\mathbf{x}_{j}^{*}\left(n_{t}+1, t_{0}\right) \text { if } n_{t}=1 \\
\mathbf{x}_{j}^{*}\left(n_{t}-1, t_{0}\right) \text { if } n_{t}=2 .
\end{array}\right.
$$

OSTBC combiner and multi-user detection can not be decoupled in the uplink due to the fact that the SCMA codewords are not multiplied by the same fading channel. Hence, a joint detection of all codewords sent by users during a space-time block should be considered. 
Spatial multiplexing: In order to achieve an improved multiplexing gain, we consider MUX-SCMA where the $N_{t}$ transmit antennas at each user are used for transmitting $N_{t}$ independent SCMA codewords. The received signal at the $n_{r}$ th antenna reads $\mathbf{y}^{n_{r}}=\sum_{n_{t}=1}^{N_{t}} \sum_{j=1}^{J} \operatorname{diag}\left(\mathbf{h}_{j}^{n_{t}, n_{r}}\right) \cdot \mathbf{x}_{j}\left(n_{t}\right)+\mathbf{n}^{n_{r}}$.

\section{REDUCED COMPLEXITY DETECTION}

\section{A. Space diversity}

In this scheme, the factor graph $\mathcal{G}_{\mathrm{SD}}$ is composed of $J$ variable nodes and $K N_{r}$ resource nodes $r_{k}$. Each variable node corresponds to a user $u_{j}$. The received signal $y_{k}^{n_{r}}$ by the $n_{r}$ th receive antenna at the $k$ th subcarrier is mapped to the resource $r_{\left(n_{r}-1\right) K+k}$ of the factor graph. For the SCMA system given in Fig. 1 the factor graph of the SD scheme having $N_{r}=2$ is given in Fig. 2(a). Both the MPA and EPA presented in Section II-B can be directly applied to $\mathcal{G}_{\mathrm{SD}}$ after replacing $h_{\left(n_{r}-1\right) K+k}^{i}$ by $\sum_{n_{t}=1}^{N_{t}} h_{k}^{n_{t}, n_{r}, i}$, where $i$ is the variable node index. The complexities of MP and EP algorithms are $\mathcal{O}\left(K N_{r} M^{d_{r}}\right)$ and $\mathcal{O}\left(K N_{r} M d_{r}\right)$, respectively.

\section{B. $O S T B C$}

In OSTBC-SCMA, the factor graph $\mathcal{G}_{\text {OSTBC }}$ is composed of $J N_{c}$ variable nodes $v_{j}$ and $T K N_{r}$ resource nodes $r_{k}$, where $N_{c}=2$ is the number of SCMA codewords transmitted during a space-time block and $T=2$ is the number of channel use time slots. Fig. 2(b) shows the factor graph of the OSTBC-SCMA scheme for the SCMA system given by the factor graph in Fig. 1. The factor graph $\mathcal{G}_{\text {OSTBC }}$ consists of two groups of variable nodes $v_{j}$, where $j \in\left\{1, \cdots, J N_{c}\right\}$. The first group $\mathcal{V}_{0}$ corresponds to the first codeword $\mathrm{x}$ sent by each user and the second group $\mathcal{V}_{1}$ corresponds to the second codeword $\tilde{\mathbf{x}}$. There are also two groups of resource nodes $r_{k}$, where $k \in\left\{1, \cdots, T K N_{r}\right\}$. The first group $\mathcal{R}_{0}$ corresponds to the subcarriers at the first channel use time slot $t_{0}$, while the second group $\mathcal{R}_{1}$ corresponds to those received in $t_{0}+1$. The complexity orders of the MP and EP algorithms are $\mathcal{O}\left(2 K N_{r} M^{2 d_{r}}\right)$ and $\mathcal{O}\left(4 K N_{r} M d_{r}\right)$, respectively. In order to apply the MP algorithm in Section II-B on the factor graph $\mathcal{G}_{\text {OSTBC}}$, the message from the resource node to the variable node given by (1) should be updated by incorporating the expressions of the received signal in OSTBC scheme given in (7) and (8). Indeed, while the same message updating given by (1) is used by the resource nodes belonging to $\mathcal{R}_{0}$, equation (1) should be modified for the resource nodes belonging to $\mathcal{R}_{1}$ as follows, according to (8): $m_{r_{k} \rightarrow v_{j}}^{(t)}(\mathbf{x})=$

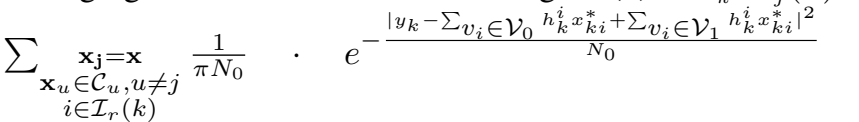
$\prod_{u \in \mathcal{I}_{r}(k) \backslash\{j\}} m_{v_{u} \rightarrow r_{k}}^{(t-1)}\left(\mathbf{x}_{u}\right)$. To apply the EPA, only step 4) in the algorithm given in Section II-B should be modified after updating the expressions of means and variances in (4) as follows. Let $r_{k}$ be a resource node corresponding to the $n_{r}$ th receive antenna and $v_{j}$ is a variable node corresponding to a user $u_{\ell}$ belonging to the group $\mathcal{V}_{p}$, where $p \in\{0,1\}$ and $\ell=\bmod (j-1, J)+1 \in \mathcal{J}$.
Result 1: The expressions of means and variances from resource nodes to variable nodes in OSTBC-SCMA are given by (9) and (10) respectively, where $n_{t}=p+1, n_{t}^{\prime}=p^{\prime}+1$, $p^{\prime} \in\{0,1\}, \ell^{\prime}=\bmod (i-1, J)+1, \bar{n}_{t}=2-p$ and $\bar{n}_{t}^{\prime}=2-p^{\prime}$.

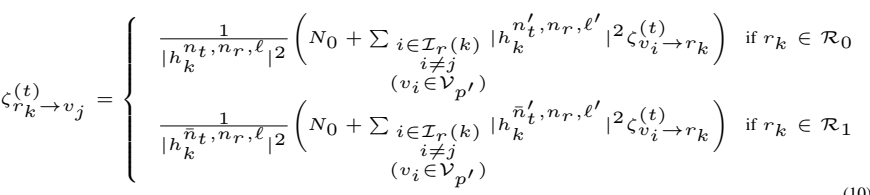

Result 1 is formulated using equations (7) and (8).

\section{MUX-SCMA}

The factor graph $\mathcal{G}_{\text {MUX }}$ is composed of $N_{t} J$ variable nodes $v_{j}$ and $K N_{r}$ resource nodes $r_{k}$, where $N_{t}=2$. The factor graph of MUX-SCMA scheme with $N_{r}=1$ is depicted in Fig. 2(c), for the SCMA system in Fig. 1. $\mathcal{G}_{\text {MUX }}$ consists of two groups of variable nodes corresponding to two codewords sent by two antennas. The complexities of the MPA and EPA are increased to $\mathcal{O}\left(K N_{r} M^{2 d_{r}}\right)$ and $\mathcal{O}\left(2 K N_{r} M d_{r}\right)$, respectively, compared to SD-SCMA scheme. It can be observed that an MUX-SCMA scheme is equivalent to a SISO-SCMA system having $N_{t} J$ users and an equivalent number of resources. Thus, both the MPA and EPA demonstrated in Section II-B can be directly applied to $\mathcal{G}_{\mathrm{MUX}}$.

\section{SIMULATION RESULTS AND DISCUSSION}

We consider an SCMA system whose factor graph is given in Fig. 1. For each of the MIMO arrangements presented in Section III, let $\rho$ be the maximum number of bits per user transmitted by two antennas during two channel time slots. For example, MUX-SCMA can transmit 4 codewords, which is equivalent to $\rho=8 \mathrm{bits} / \mathrm{user} / 2$ transmit antennas $/ 2$ channel use periods, when a codebook of size $M=4$ codewords is employed. Furthermore, the bit error rate (BER) performance is studied for a fixed value of $\rho$ in all schemes in order to enable fair comparison. SCMA codebooks are designed according to the method proposed in [8]. We use damping to improve the performance of EPA.

Figure 3(a) depicts the uncoded BER performance curves of the SD, OSTBC and MUX systems, where $N_{r}=1$ and $\rho=4$. The MPA is employed with 6 iterations. It can be observed that a BER gain of nearly $4 \mathrm{~dB}$ is obtained using OSTBC-SCMA with respect to MUX-SCMA, at BER $=10^{-5}$. Moreover, it can be seen that OSTBC-SCMA outperforms SDSCMA by $3 \mathrm{~dB}$ at BER $=10^{-5}$. To further demonstrate the efficiency of OSTBC-SCMA, we also consider a space-time code (STC) scheme based on both space and time diversity, which is obtained after removing the minus sign and the conjugate operators in the encoding matrix (6). Figure 3(a) shows that OSTBC-SCMA exhibits a better BER performance than STC-SCMA at the same complexity of detection. This demonstrates the ability of OSTBC-SCMA for improving the reliability of transmission although the OSTBC combiner and signal decoding are no more separable. 
(a)

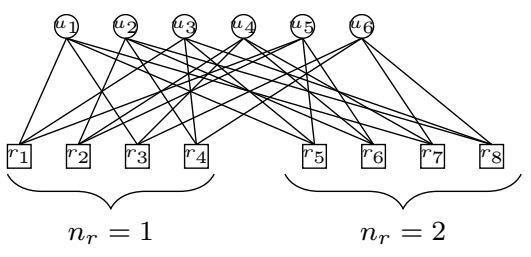

(b)

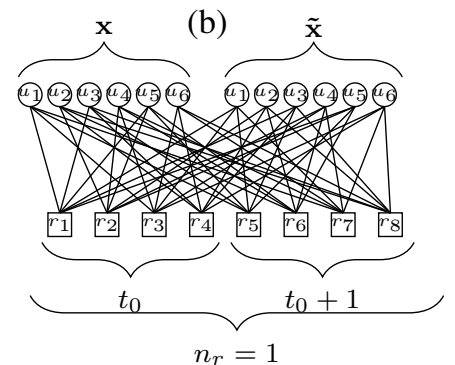

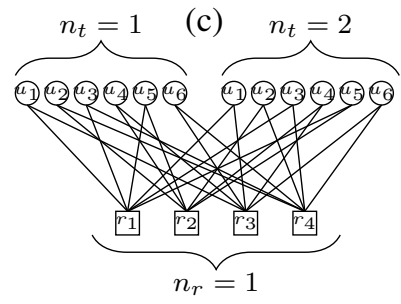

Figure 2. Factor graph representations of (a) SD-SCMA with $N_{r}=2$, (b) OSTBC-SCMA with $N_{r}=1$, (c) MUX-SCMA with $N_{r}=1$.

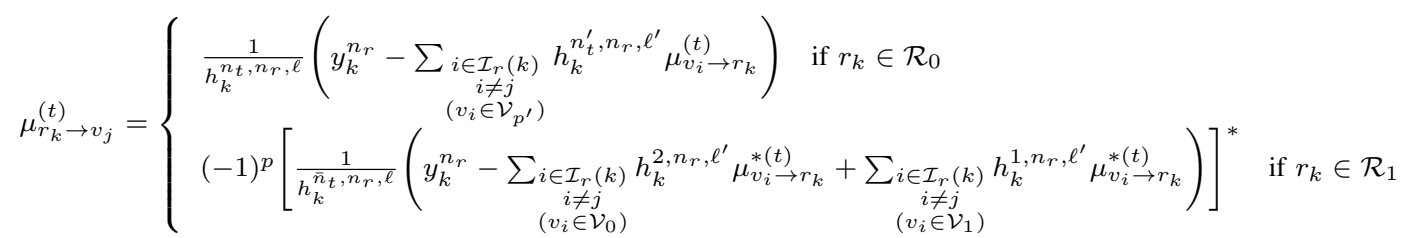
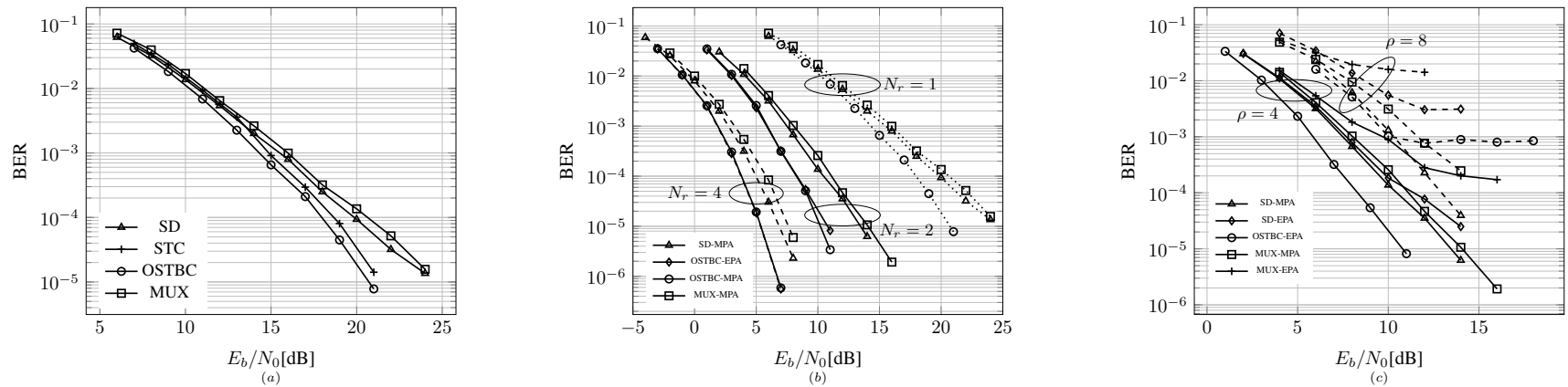

Figure 3. (a) BER performance using MPA. $\rho=4, N_{r}=1$. (b) Effect of $N_{r}, \rho=4$. (c) Comparison for different values of $\rho$. $N_{r}=2$

Figure 3(b) shows the improvement in the BER performance for the OSTBC, SD and MUX schemes, as the number of receive antennas increases. The BER performance for OSTBCSCMA scheme is portrayed for both MPA and EPA, except when $N_{r}=1$, where the EPA suffers from high error floor at $\mathrm{BER}=10^{-2}$. It can be seen that the performance of EPA is close to that of MP, especially when $N_{r}$ increases. This indicates that by adding more receive antennas the low complexity EPA detector can be used more efficiently.

Figure 3(c) depicts the BER performance of uncoded MIMO-SCMA, when $N_{r}=2$, at different values of $\rho$. The BER performances of SD-SCMA and MUX-SCMA are given for both the MPA and EPA algorithms. However, the BER performance of OSTBC-SCMA is given only for the EPA, since the complexity of MPA increases with the codebook size. It can be observed that EPA may exhibit high error floor depending on the codebook size and on the number of receive antennas. The problem of error floor in EPA may be solved using channel coding.

\section{CONCLUSION}

We have studied various uplink MIMO-SCMA schemes and investigated low-complexity detection algorithms based on EPA. We analyzed the performance of the EPA based on the
MIMO-SCMA scheme, the codebook size $M$ and the number of receive antennas $N_{r}$. Results showed that the performance of EPA improves when $M$ decreases and $N_{r}$ increases.

\section{REFERENCES}

[1] H. Nikopour and H. Baligh, "Sparse code multiple access," in IEEE 24th Annual International Symposium on Personal, Indoor, and Mobile Radio Communications (PIMRC), Sept 2013, pp. 332-336.

[2] D. N. C. Tse, P. Viswanath, and L. Zheng, "Diversity-multiplexing tradeoff in multiple-access channels," IEEE Transactions on Information Theory, vol. 50, no. 9, pp. 1859-1874, Sept 2004.

[3] W. Yuan, N. Wu, Q. Guo, Y. Li, C. Xing, and J. Kuang, "Iterative receivers for downlink MIMO-SCMA: message passing and distributed cooperative detection," IEEE Transactions on Wireless Communications, vol. 17 , no. 5, pp. 3444-3458, May 2018.

[4] S. M. Alamouti, "A simple transmit diversity technique for wireless communications," IEEE Journal on Selected Areas in Communications, vol. 16, no. 8, pp. 1451-1458, Oct 1998.

[5] S. Han, C. Guo, W. Meng, C. Li, Y. Cui, and W. Tang, "The uplink and downlink design of MIMO-SCMA system," in International Wireless Communications and Mobile Computing Conference (IWCMC), Sept 2016, pp. 56-60.

[6] X. Meng, Y. Wu, Y. Chen, and M. Cheng, "Low complexity receiver for uplink SCMA system via expectation propagation," in IEEE Wireless Communications and Networking Conference (WCNC), March 2017.

[7] I. A. Hemadeh, M. El-Hajjar, and L. Hanzo, "Hierarchical multifunctional layered spatial modulation," IEEE Access, vol. 6, pp. $9492-$ 9533, 2018.

[8] Z. Mheich, L. Wen, P. Xiao, and A. Maaref, "Design of SCMA Codebooks Based on Golden Angle Modulation," IEEE Transactions on Vehicular Technology, vol. 68, no. 2, pp. 1501-1509, Feb 2019. 